\title{
Superhydrophobic, Hybrid, Electrospun Cellulose Acetate Nanofibrous Mats for Oil/Water Separation by Tailored Surface Modification
}

\author{
Osman Arslan, ${ }^{* \dagger}$ Zeynep Aytac, $^{\dagger, \ddagger}$ and Tamer Uyar, ${ }^{* \dagger, \dagger}$ \\ ${ }^{\dagger}$ UNAM-National Nanotechnology Research Center, Bilkent University, 06800, Ankara, Turkey \\ ${ }^{\ddagger}$ Institute of Materials Science \& Nanotechnology, Bilkent University, Ankara, 06800, Turkey \\ Supporting Information
}

ABSTRACT: Electrospun cellulose acetate nanofibers (CA-NF) have been modified with perfluoro alkoxysilanes (FS/CA-NF) for tailoring their chemical and physical features aiming oil-water separation purposes. Strikingly, hybrid FS/CA-NF showed that perfluoro groups are rigidly positioned on the outer surface of the nanofibers providing superhydrophobic characteristic with a water contact angle of $\sim 155^{\circ}$. Detailed analysis showed that hydrolysis/ condensation reactions led to the modification of the acetylated $\beta(1 \rightarrow 4)$ linked D-glucose chains of CA transforming it into a superhydrophobic nanofibrous mat. Analytical data have revealed that CA-NF surfaces can be selectively controlled for fabricating the durable, robust and water resistant hybrid electrospun nanofibrous mat. The $-\mathrm{OH}$ groups available on the $\mathrm{CA}$ structure allowed the basic sol-gel reactions started by the reactive FS hybrid precursor system which can be monitored by spectroscopic analysis. Since alkoxysilane groups on the perfluoro silane compound are capable of reacting for condensation together with the CA, superhydrophobic nanofibrous mat is obtained via electrospinning. This structural modification led to the facile fabrication of the novel oil/water nanofibrous separator which functions effectively demonstrated by hexane/oil and water separation experiments. Perfluoro groups consequently modified the hydrophilic CA nanofibers into superhydrophobic character and therefore FS/CA-NF could be quite practical for future applications like water/oil separators, as well as self-cleaning or water resistant nanofibrous structures.

KEYWORDS: oil/water separation, superhydrophobic, electrospinning, nanofibers, cellulose acetate, perfluorosilane, sol-gel

\section{INTRODUCTION}

As one of the most peaked nanofiber production technique in the recent years, electrospinning has been extensively investigated for the fabrication of polymeric or composite nanofibers exhibiting high surface area-to-volume and lengthto-diameter ratios for diverse applications. ${ }^{1-5}$ Since polymeric systems allow the various practical applications, such as filtration, separation, membranes technologies, wound dressing applications, and sensing implementations by blending or modifying the basic skeleton with another component for the electrospinning applications, one can produce tailored nanocomposite hybrid fibers. ${ }^{6-11}$ For a standard electrospinning method, polymer solutions, blends, or hybrid mixtures are placed in a container (generally into a syringe in laboratory scale applications) having different size and features and a strong electrostatic force is applied. Capillary conditions help to the polymeric solution which will be ejected from the syringe under high electrical field and collected as nonwoven nanofibrous material on the collector. ${ }^{12-19}$ First, electrospinning parameters are optimized for the mixture then typically submicron range fibers are electrospun and therefore they are named as nanofibrous mats or ultrafine fibers. It is widely known that cellulose acetate (CA) has an important place among the polymer applications for the electrospinning of permeable filtration systems, membranes, reverse osmosis or aerosol treatments. $^{5-7}$

Reason of the wide utilization characteristics of the CA can be attributed to its low absorption characteristics, thermal stability, easy to modify feature and physical durability. Strikingly electrospun nanofibrous mats of CA provide extremely chemical resistant platforms against most common organic solvents and additionally stable at very diverse $\mathrm{pH}$ values ranging from 3 to $12 .^{20,21}$

For the modification of the CA nanofibrous mats, some specific grafting or modifying agents, such as glycidyl methacrylate-g-PEG for organic fouling prevention, ${ }^{22}$ vinyltrimethoxysilane for selective gas permeation with $\mathrm{CO}_{2}$ and $\mathrm{H}_{2} \mathrm{~S}$ having reduced brittleness, ${ }^{23}$ and poly[(ar-vinylbenzyl)trimethylammonium chloride)] [poly(VBTAC)] for the reusable nanomats by measuring the adsorption capacity

Received: May 6, 2016

Accepted: July 11, 2016

Published: July 11, 2016 
targeting the separation of DNA, were used. ${ }^{24}$ Additionally 2hydroxyethyl methacrylate (HEMA), 2-dimethylaminoethyl methacrylate (DMAEMA) and acrylic acid for efficient supports for enzyme immobilization, ${ }^{25}$ poly $(N$-isopropylacrylamide) for the responsive superhydrophobic electrospun nanofibrous mats, $\mathrm{ZnO}$ nanoparticles for optical properties have been studied and deeply analyzed. As expected $-\mathrm{OH}$ anchor groups act as the modification points but additionally ester groups or double bonds can be also used. ${ }^{26}$

Superhydrophobic nanomaterials recently gained a huge interest due to their oil adsorption and separation features. If the chemical nanostructure is carefully designed or surface is modified as desired with hydrophobicity providing molecules, final nanomaterial may show the oil/water separation character. Since oil phase spread easily and penetrate via natural pores on the nanomaterial, while water phase stay on the surface and repelled. In the literature, construction of rough surfaces on a hydrophobic surface or surface modifiers with low surface energy methodologies can be used. Since core/shell structures, aerogel hybrid materials, selectively rough surfaces with certain morphologies are used, we have designed a route for electrospun superhydrophobic CA nanomats which can be utilized in the oil/water separation applications.

Hence, in present study we have developed a facile method for the fabrication of the superhydrophobic CA electrospun nanofibrous mats for the oil/water separation systems. There are many new nanomaterials for the oil/water separation in the very recent literature providing vast information about how the surface modification, copolymerization, $\mathrm{pH}$ sensitivity, alkyl groups or starting inorganic materials can affect the real applications. ${ }^{27-34}$ As a new approach, we have functionalized the electrospun CA nanofibrous mats with $1 \mathrm{H}, 1 \mathrm{H}, 2 \mathrm{H}, 2 \mathrm{H}$ Perfluorooctyltriethoxysilane (FS) via hydrolysis and condensation reactions to obtain FS/CA-NF. It has been observed that organic solvent systems allowed the functionalization of the $\mathrm{CA}$ surface via $-\mathrm{OH}$ groups and superhydrophobic, bead free nanofibrous mat has been obtained. For the practical application, we have shown that electrospun, superhydrophobic FS/CA-NF mat can be used for oil/water separation. The most salient feature of our work is its unique utilization of the FS as surface modifier, its proper attachment onto the electrospun nanofibrous mat surface by well programmed curing and its useful application performance exemplified with oil/water separation experiments.

\section{EXPERIMENTAL SECTION}

2.1. Electrospinning Cellulose Acetate. (CA, $M_{w}=30000 \mathrm{~g} /$ mol, $39.8 \mathrm{wt} \%$ acetyl, Sigma-Aldrich) dichloromethane (DCM, $\geq 99 \%$ (GC), Sigma-Aldrich), acetone ( $\geq 99.7 \%$ (GC), Sigma-Aldrich) have been used. $1 H, 1 H, 2 H, 2$ H-Perfluorooctyltriethoxysilane (Dynasilan F8261 was kindly donated from Evonik Industries (Germany). Solution of the $15 \% \mathrm{CA} /($ dimethylacetamide(DMAc):acetone) was prepared and homogenized by stirring which was followed by the addition of FS $(\mathrm{w} / \mathrm{w}, 100 \mu \mathrm{L})$ as in the form of a mixture with $5 \mu \mathrm{L}$ of $0.1 \mathrm{M} \mathrm{HCl}$. DMAc:Acetone was used as (w/w) 1:2 mixture. Homogeneous FS solutions were obtained after $1 \mathrm{~h}$ stirring with magnetic stirrers. Prepared CA-FS mixtures have been loaded into the $3 \mathrm{~mL}$ syringe and positioned horizontally on the syringe pump (Model KDS $101 \mathrm{KD}$ scientific). The electrode of the high voltage power supply (Matsusada Precision, AU Series) was clamped to the metal needle tip and the target aluminum collector was grounded. The electrospinning of these hybrid solutions was performed generally at the following parameters: applied voltage $15 \mathrm{kV}$, tip-to-collector distance $15 \mathrm{~cm}$ and the solution flow rate $0.5 \mathrm{~mL} / \mathrm{h}$. CA-FS mixtures were deposited on a grounded stationary metal collector covered by a piece of aluminum foil. The electrospinning apparatus was enclosed in a Plexiglas box and the electrospinning was carried out at $25{ }^{\circ} \mathrm{C}$ at $30 \%$ relative humidity. Curing and cross-linking of the nanofibrous mats have been achieved at $130{ }^{\circ} \mathrm{C}, 30 \mathrm{~min}$ conditions.

2.2. Measurements and Characterization. The atomic composition investigation (EDX mapping) of the pristine $\mathrm{CA}$ and FS modified nanofibrous CA mats (FS/CA-NF) have been performed together with morphology and the diameter analyses using a scanning electron microscope (SEM) (Quanta 200 FEG, FEI). Nanofibrous mats were coated with the $5 \mathrm{~nm} \mathrm{Pt} / \mathrm{Au}$ (with PECS-682) prior to the SEM imaging. Average diameters of the hybrid nanofibrous mats were calculated by counting and measuring 100 nanofibrous mat diameters and plotting them with respect to their frequencies. Nonlinear fitting on the obtained nanofibrous mat diameters gave an average value with a calculated statistical standard deviation and error bars. The static water contact angles on the hybrid nanofibrous mats were evaluated using contact angle analyzing instrument (OCA30, Dataphysics Instrument Company) at room temperature. Deionized water (0.4 $\mu \mathrm{L}$ ) was automatically dropped on the nanofibrous mats on glass surfaces and Laplace-Young fitting was applied on contact angle measurements. The measurements were repeated 5 times at different positions of the FS/CA-NF mat for an average value. The surface and atomic composition of nanofibrous mat was performed by X-ray photoelectron spectroscopy (XPS, Thermo Scientific). XPS spectra have been obtained by a flood gun charge neutralizer system equipped with a monochromated $\mathrm{Al} \mathrm{K \alpha} \mathrm{X}$-ray source $(h \nu=1486.6 \mathrm{eV})$ from $400 \mathrm{~mm}$ spot size on the nanofibrous mat. For the proton nuclear magnetic resonance $\left({ }^{1} \mathrm{H}\right.$ NMR) measurements deuterated chloroform $\mathrm{CDCl}_{3}-\mathrm{D}_{1}$ (Merck) has been used as reference NMR solvent. Molecular structure and interactions between the precursors were examined by ${ }^{1} \mathrm{H}$ - NMR, Bruker D PX-400 and samples have been analyzed after dissolving in $\mathrm{CDCl}_{3}-\mathrm{D}_{1}$. The spectra have been recorded at $400 \mathrm{MHz}$ and 16 total scan applied. TGA analyses (Q500, TA Instruments) were performed to demonstrate the availability of each components together with CA and sample analyses were carried out from 75 to $500{ }^{\circ} \mathrm{C}$ at $20^{\circ} \mathrm{C} / \mathrm{min}$ heating rate and $\mathrm{N}_{2}$ was used as a purge gas. The infrared spectra of the nanofibrous mats were obtained by using a Fourier transform infrared spectrometer (FTIR) (BrukerVERTEX 70).For measurement, the samples were mixed with potassium bromide $(\mathrm{KBr})$ and pressed as pellets. The scans (64 scans) were recorded between 4000 and $400 \mathrm{~cm}^{-1}$ at a resolution of 4 $\mathrm{cm}^{-1}$.

2.3. Separation Experiments. For the oil/water separation experiments oil $/ \mathrm{H}_{2} \mathrm{O}$ phases were prepared. For the sustainability final FS/CA-NF composition has been prepared as in the $\mathrm{KBr}$ pellet preparation to maintain the nanomats mechanical integrity. Prepared nanofibrous mat can also be used in other forms. Same amount of water and oil phases, colored with methylene blue (water) and yelloworange (4-amino azo benzene-oil absorb oil phase and squeezed into an empty vessel for absorbed oil to be released. Repetition and different mixtures have been utilized for showing the effectiveness of the nanofibrous mats.

\section{RESULTS AND DISCUSSION}

For the fabrication of perfluoro modified electrospun CA nanofibrous mats (FS/CA-NF), available $-\mathrm{OH}$ groups on $\mathrm{CA}$ act as remarkable active centers to tailor the final materials. ${ }^{12,13}$ It is widely known that sol-gel reactions propagate by the activation of the alkoxysilane moieties. Chemical structure of the modifying alkoxy groups on the silanes have the affinity to the exchange reactions which can be started by an attack of reactive group via basic or acidic catalysis. After that, through hydrolysis/condensation reactions, removal of water and alcohol facilitates the reactions. ${ }^{35,36}$ Due to the chemical structure and abundant nature, cellulose is widely used industry in many different areas with its natural or acetylated form. Therefore, CA contains $-\mathrm{OH}$ and $\mathrm{COOCH}_{3}$ (acetate) groups on the $\beta(1 \rightarrow 4)$ linked D-glucose chain (Figure 1 and Figure 

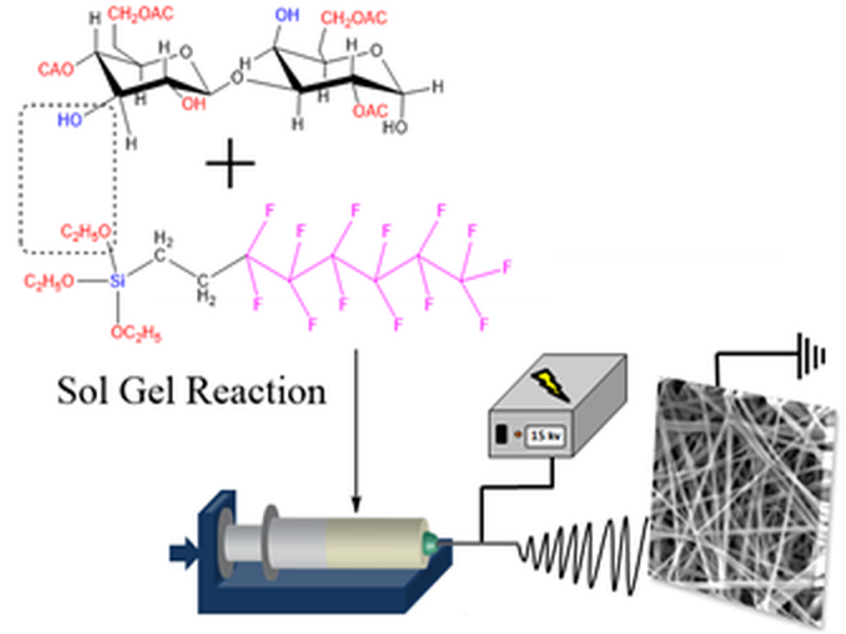

Figure 1. Electrospinning setup for the fabrication of superhydrophobic perfluoro-modified cellulose acetate nanofibrous mats (FS/CA-NF).

2a). During sol-gel reactions of this carbohydrate chain, alkoxysilanes react with $-\mathrm{OH}$ groups which is close to the $\mathrm{Si}$ center and perfluoro groups align toward outer side in the

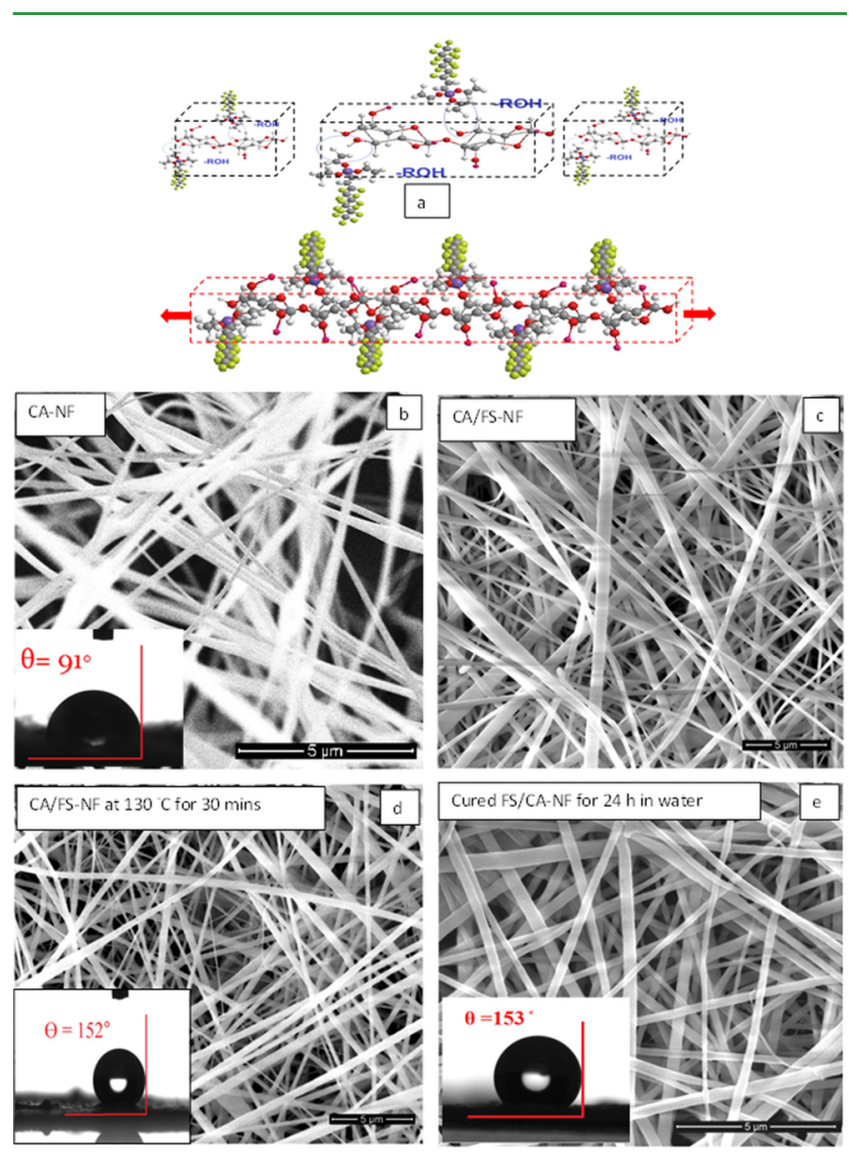

Figure 2. (a) Superhydrophobic modification of CA polymer with FS precursor via hydrolysis/condensation reactions (b) Electrospun CANF (inset is water contact angle measurement. (c) Superhydrophobic FS/CA-NF before and (d) after the heat curing $\left(130^{\circ} \mathrm{C}\right.$ for $30 \mathrm{~min}$, inset is water contact angle measurement). (e) Cured superhydrophobic FS/CA-NF after $24 \mathrm{~h}$ in water, contact angle measurement is given as an inset figure. space. This effect has also been observed with nanoparticles and denoted as "Umbrella Effect" providing an extreme superhydrophobic character. ${ }^{37,38}$ It is widely known that, when alkoxysilanes react with the $-\mathrm{OH}$ groups, they can also attach other effective functionalities like $-\mathrm{NH}_{2},-\mathrm{SH}$, epoxy, alkyl, $-\mathrm{COOH},-\mathrm{Cl}$. On the basis of these fundamental properties, electrospinning nanofibrous mat preparation perspective has been applied to donate the perfluoro groups on the CA hybrid nanofibrous mats. ${ }^{26}$ CA was reacted with FS (Figure 1) according to the ratio of the $\mathrm{CA}$ solid content in total formulation. For the preparation of the electrospinning solution, FS must have been added into the CA solution in the early stages of the mixing as superhydrophobic modifier for the sol-gel reactions. FT-IR spectra (Figure S1) and ${ }^{1} \mathrm{H}$ NMR analyses (Figure S2) showed that hydrolysis/condensation reactions led to the modification of the acetylated $\beta(1 \rightarrow 4)$ linked D-glucose chains showing superhydrophobic character with higher than 150 degree water contact angles (Figure 2d).

During preparation, CA solution was stirred about $2-3 \mathrm{~h}$ within the solvent system and FS has been introduced together with $\mathrm{H}_{2} \mathrm{O}(0.1 \mathrm{M} \mathrm{HCl}) . \mathrm{H}_{2} \mathrm{O}$ is necessary to start the hydrolysis and condensation reactions for the $\mathrm{CA}$ modification. Since organic solvents are available, amphiphilic nature of the FS precursor should be sensitized by an organic/inorganic mixture. Therefore, water containing mixture provides a very suitable environment for the alkoxysilane which also allows the beadfree, superhydrophobic hybrid electrospun nanofibrous mats. After electrospinning of the hybrid precursor mixture, collected nanofibrous mats have been cured at $130{ }^{\circ} \mathrm{C}$ for about $30 \mathrm{~min}$. This is necessary for the completion of condensation reactions since intermediate structures or alkoxy groups may be in an uncompleted form. Figure $2 \mathrm{c}$ and $2 \mathrm{~d}$ represents the SEM images of FS/CA-NF before and after curing which their fiber diameter averages were given also at Figure S3. As presented in the figures, the average diameter of the thermally cured FS/CANF nanofibers did not change; before curing, nanofibers had $290 \pm 110 \mathrm{~nm}$ in diameter and after curing it was recorded as $280 \pm 105 \mathrm{~nm}$. Yet, cured nanofibers showed superhydrophobic character with the values presented in Figure S4 with 5 different contact angle between $\sim 150-155$ degree. CA$\mathrm{NF}$ showed 91 degree contact angle (Figure $2 \mathrm{~b}$ inset) which is compatible with the literature values. ${ }^{25}$ FS/CA-NF have been compared with non cured and CA-NF after water immersion (Figure 3a) during $24 \mathrm{~h}$ as templated in Figure 2e. According to the SEM results of pristine CA-NF have the diameter of $850 \pm$ $230 \mathrm{~nm}$ which was increased to the $1255 \pm 470 \mathrm{~nm}$ after water immersion (Figure S5). This clearly showed that CA-NF absorbed water and swelling features of the CA-NF was also presented in Figure S6. Interestingly, cured FS/CA-NF showed no water absorption. Results therefore showed that FS modification on the nanofibrous mats provided an umbrella effect and prevented the water interaction. Hence, contact angle (Figure 2d inset and 2e inset), EDX mapping (Figure 3b), XPS analysis (Figure 4) and ${ }^{1} \mathrm{H}$ - NMR results (Figure S2) revealed that perfluoro groups are on the surface and water penetration into the FS/CA-NF mat is hardly possible. When SEM image of the FS/CA-NF after $24 \mathrm{~h}$ immersion is analyzed (Figure 2e), it is very clear that there is no variation in the nanofiber morphology or diameter. Oppositely, results for pristine CANF (Figure 2b, Figure S5) clearly showed that under normal conditions water swelling is quite visible and a remarkable increase in fiber diameter was detected. At least 63\% increase in diameter was detected which can vary with different structural, 

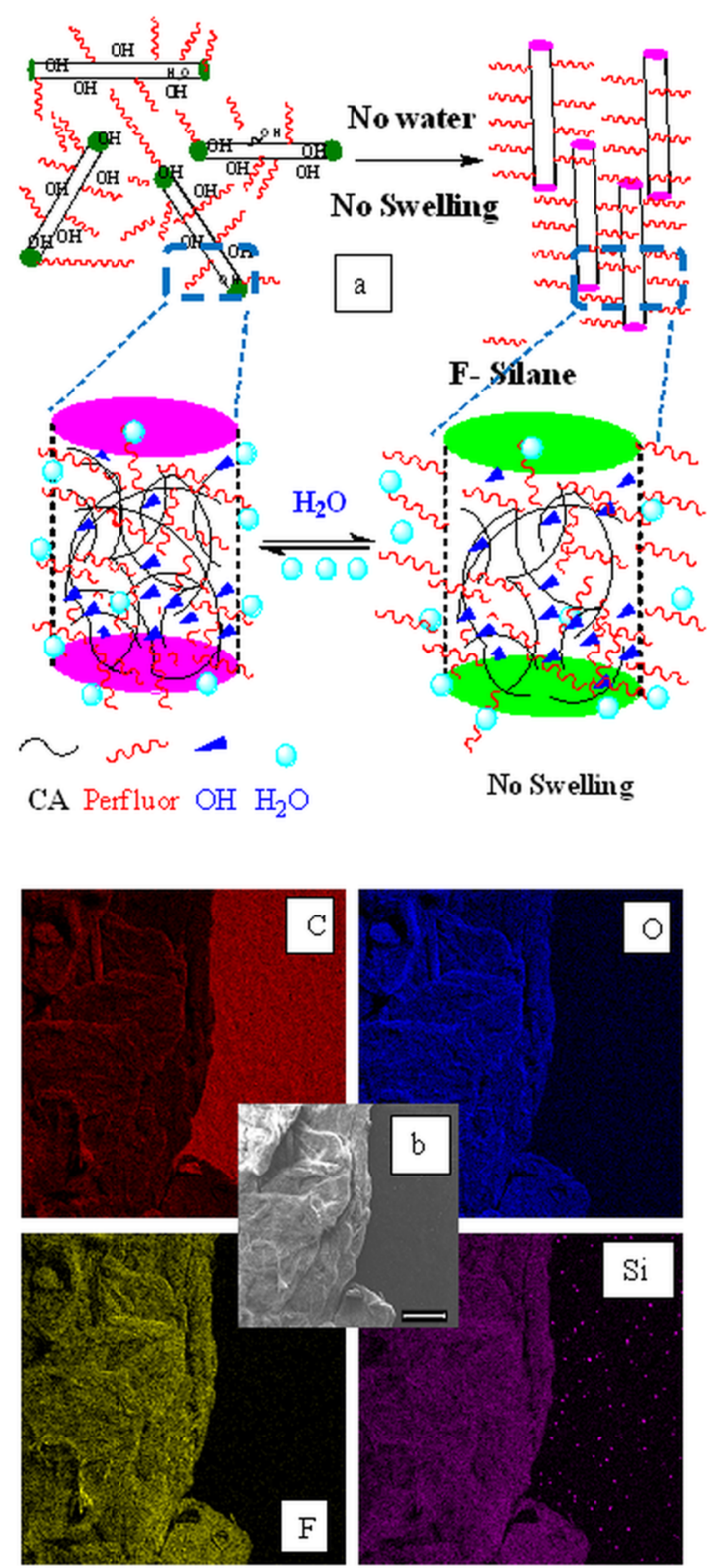

Figure 3. (a)Schematic representation of FS/CA-NF and $\mathrm{H}_{2} \mathrm{O}$ interaction and alignment of the perfluoro groups restricting water penetration. (b) SEM image of FS/CA-NF (center image) (scale bar = $100 \mu \mathrm{m})$ and atomic $(\mathrm{C}, \mathrm{O}, \mathrm{F}$, and $\mathrm{Si}$ ) mapping of $\mathrm{FS} / \mathrm{CA}-\mathrm{NF}$ by EDX.

temperature and modifications values (Figure S5). When FS/ CA-NF are immersed into the water $24 \mathrm{~h}$, expected hydrogen bonding interaction enhancing the stacking behavior and causing entanglement was not observed. In the pristine CANF after water swelling process, swelling and slight attachments of nanofibers one to another is visible in SEM images (Figure S5a). EDX atomic mapping of CA-NF have been presented in Figure S7 showing clear availability of $\mathrm{C}$ and $\mathrm{O}$. Together with SEM results, EDX analysis (Table S1, Figure 3b) interestingly and strikingly revealed that, perfluoro groups does not allow the water penetration and FS/CA-NF do not change their physical appearance and morphology as swelling equations below also highlighted

$$
Q=W_{2} W_{1} / W_{1}
$$
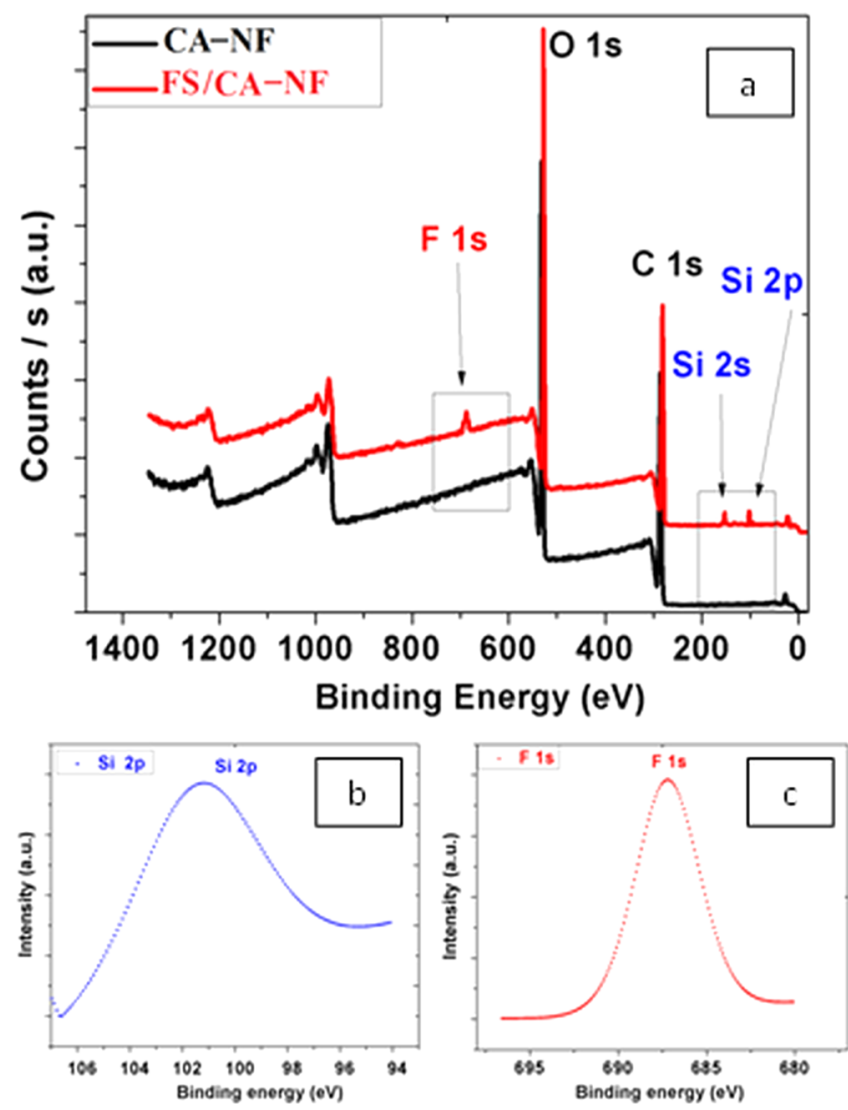

Figure 4. (a)XPS survey analysis for the final cured material and (b) high-resolution deconvoluted peaks for $\mathrm{Si} 2 \mathrm{p}$ and (c) for F 1s cores.

$$
R=T_{\mathrm{w}} / T_{\mathrm{d}}
$$

where $Q$ is the absorbency, $W_{1}$ is the initial weight, and $W_{2}$ is the weight of the nanofibrous mat when it is wet and results showed that CA-NF gained weight after $24 \mathrm{~h}$. But FS/CA-NF did not show any visible weight variation. Additionally in eq 2 , where $R$ is related with $T_{\mathrm{w}} / T_{\mathrm{d}}$ showing the wet-to-dry ratio for thickness, $T_{\mathrm{w}}$ is the thickness of the sample after maximum water absorption, $T_{\mathrm{d}}$ is the thickness of the dry nanofiber sample, and results showed in the basis of nanofibrous mat thickness that $R$ does not have any meaningful result since there is no diameter change for the FS/CA-NF. Results (Figure S6) showed that $43 \%$ increase in the weight for CA-NF is observed. Oppositely, surface alignment of the perfluoro groups on the FS/CA-NF can be detected by EDX mapping as shown in Figure $3 \mathrm{~b}$. Figure $3 \mathrm{a}$ structurally and morphologically imitates the FS/CA-NF and their interaction with the water is anticipated. Image shows that perfluoro groups protect the surface and decrease the water penetration due to the superhydrophobic behavior and umbrella effect. Additionally possible hydrogen bonding centers were not able to form hydrogen bonding or water swelling was not possible which could have increased the average diameter of FS/CA-NF by water loading. Structurally when $-\mathrm{OH}$ containing nanofibers are immersed into the water, hydrogen bonding is expected. EDX mapping results (Figure $3 b$ ) with ${ }^{1} \mathrm{H}$ NMR spectra (Figure S2) showed that $\mathrm{F}$ atoms are remarkably visible facilitating the water repelling character of the nanofibrous mats. According to the XPS survey (Figure 4a) results, $F$ peaks together Si peaks appear at $688.1 \mathrm{eV}$ for the F $1 \mathrm{~s}$ and 101.6 and $154.2 \mathrm{eV}$ respectively for $\mathrm{Si} 2 \mathrm{p}$ and $\mathrm{Si} 2 \mathrm{~s}$ in the XPS survey. 

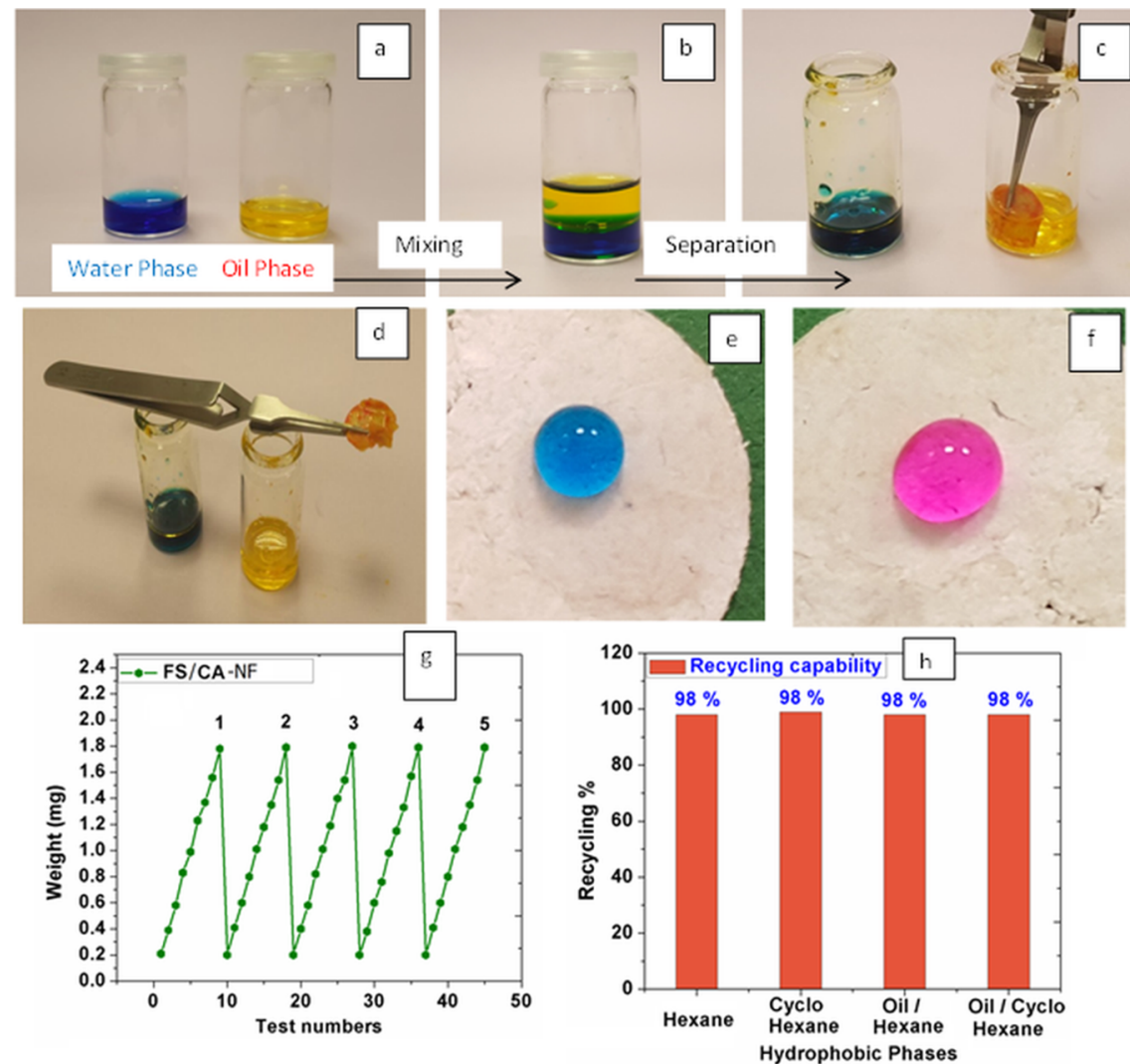

Figure 5. (a) Oil/hexane and water phases before separation. (b) Mixed oil/water mixture. (c) Separated phases by nanofibrous mat structure. (d) No MB adsorption for FS/CA-NF after separation. (e, f) Water droplets on FS/CA-NF mat. (g) Repeatability of the separation experiment and (h) separation yields for different mixtures.

Deconvoluted high resolution XPS spectra for the F 1s and Si $2 \mathrm{p}$ cores have also been presented in Figure $4 \mathrm{~b}$ and $4 \mathrm{c}$. These peaks very clearly absent in the pristine CA-NF survey spectra when compared to the FS/CA-NF. In addition to that atomic variations, calculated amounts of each atom were compatible with the results obtained from EDX analysis. Consequently, XPS results showed that perfluoro groups are on the nanofibrous mat surfaces. Moreover, according to the ${ }^{1} \mathrm{H}$ NMR analysis ethoxy groups on the FS have been attached onto the CA polymer chain in the final mixture which will be condensed after heat treatment. Additionally $\mathrm{H}$ atoms connected to the $\mathrm{C}$ atom which is between $\mathrm{CF}_{2}$ groups and $\mathrm{Si}$ atom have been observed at $0.92 \mathrm{ppm}\left(\mathrm{Si}-\mathrm{CH}_{2}-\right)$ and at 2.2 ppm $\left(\mathrm{Si}-\mathrm{CH}_{2}-\mathrm{CH}_{2}-\right)$ as presented. Additionally acetyl ( $\mathrm{COCH} 3)$ group attached to the $\mathrm{CH}_{2}-\mathrm{O}-$ and other available $\mathrm{H}$ atoms in $-\mathrm{OH}$ or $\mathrm{CH}_{2}-$ position at the $\beta(1 \rightarrow 4)$ linked Dglucose structure have been denoted in the ${ }^{1} \mathrm{H}$ NMR spectra. Since electrospun 1-D nanofibrous mats can also be controlled in the molecular level by different methods, high surface-tovolume ratio, selective pore size and possible cross-linking with heat and UV-curing capability features, unusual adsorption or luminescent material fabrication is possible from these new class of nanomaterials. ${ }^{39-41}$ For investigating the thermal character, we have conducted the TGA analysis (Figure S8) and results showed that attached FS part is clearly visible in the final material together with the CA decomposition until 225$250{ }^{\circ} \mathrm{C}$ where the organic backbone starts to decompose. Since heat treatment was used for better sol-gel reactions, early decomposition stages for the FS pattern in TGA is clear and differences in thermogram of the hybrid FS/CA-NF is easily noticeable and comparable with previous works. ${ }^{42} 26 \%$ of the total material weight has been removed until $250{ }^{\circ} \mathrm{C}$ but the volatile solvents or adsorbed water can also be evaporated until this temperature. CA backbone is clearly visible in the TGA graph which composes almost $68 \%$ of the system.

As widely known superhydrophobic-superoleophilic nanomaterials are natural and typical oil removing materials since superhydrophobicity provides that oil phase spread easily, adsorbed and penetrate via natural pores on the nanomaterial while water phase is repelled. This basic phenomena separates oil from an oil/water mixture. Hence, surface hydrophobicity or wettability determined by surface chemical composition and morphological/topographical structure provided by fine chemical tuning is important for superhydrophobic-superoleophilic materials. Generally two methods can be applied namely constructing a rough structure on a hydrophobic surface or using surface modifying chemicals with low surface energy on a rough surfaces. ${ }^{43}$ Previously different morphologies such as core/shell, aerogel structures, and selectively rough surfaces have been showed for different applications after cellulosic modifications. ${ }^{44-47}$ Additionally materials as the separator or oil removing material such as nanofibrous mat-based materials, metallic mesh-based materials, carbon and its derived materials and particles have also been studied. Different materials can be used for oil/water separation such as porous metal films, fabric materials, soft and flexible organic materials such as electrospun nanofibrous mats since prepared nanowebs can provide microscale roughness and their native porosity ensures the free passage of anticipated liquids. ${ }^{4-53}$ As presented in the Figure 5 water-oil/mixtures was prepared and electrospun 
superhydrophobic FS/CA-NF was used as squeezable mats to remove the oil phase from water. For clarity methylene blue and 4-amino azobenzene have been dissolved in the water and oil phase, respectively. For the oil-water separation experiments oil/hexane (4:1)-water mixtures have been prepared. Electrospun nanofibrous mats have been detached from the $\mathrm{Al}$ foil and brought together into a tablet form. Utilization of the nanofibrous electrospun mats is technically hard for our designed separation experiment and it was observed that electrospun mat comes apart after dipping process and tablet form is more convenient. Additionally, maintaining the mechanical integrity of the nanofibrous mat during the experiments was succeeded with this route. Electrospun nanofibrous mat has been simply dipped into this prepared (Figure $5 \mathrm{a}-\mathrm{c}$ ) oil/water mixture and then oil part was removed by squeezing absorbed oil on the network (Figure 5c). A short video showing this procedure was presented in Video 1S. Same experiment was conducted with non-modified CA-NF as shown in Figure S9 revealing aqueous $\mathrm{MB}$ blue adsorption on the nanofibers. By this simple and fast method we could remove hexane, cyclohexane and their 4:1 oil mixtures very rapidly as shown in Figure 5c. According to the measurements, nanofibrous mat can absorb almost 3 fold more solvent/oil as compared to its normal weight as measured and observed. Additionally since there is no $\mathrm{MB}$ adsorption (Figure $5 \mathrm{~d}$ ) on the surface it can be easily noticed that water is highly repelled during separation (Figure 5e and f). Since electrospun, hybrid material exhibit high volume-based absorption capacities, we can simply assume that almost all of its volume is used for oil storage additionally with its natural internal pores. Obtained electrospun, hybrid sponge can also be reapplied for oil-water separation for many cycles (Figure $5 \mathrm{~g}$ ) and for other mixtures (Figure $5 \mathrm{~h}$ ). Intermediate points in $6 \mathrm{~g}$ are the weight values corresponding to the transferred oil portions. When we dip and adsorb the oil part from the mixture $\left(W_{1}=\right.$ mat + oil $)$ and squeeze it to release the oil $\left(W_{2}=\right.$ mat $)$, basically we transfer small portions of the oil into other vessel. Quantitative amounts of oil portions were detected during the separation by weight observation of the mats after the oil adsorption and oil release. Sometimes this small portion is not perfectly same at every adsorption/squeezing cycle but total separation was completed generally with only 2 or 3 additional cycle. These portions were inversely plotted to show the stepwise oil removal in each case. This experiment showed the enhanced recyclability of the hybrid structure of the perfluoro modified nanofibrous mats.

As a summary, we are presenting a novel electrospun, surface modified CA nanofibrous mat allowing the formation of the superhydrophobic nanofibrous materials with excellent separation efficiencies. Obtained FS/CA-NF mats may have high potentials in different applications such as spilled oil cleaning and oil/water separations.

\section{CONCLUSION}

In this Research Article, we have fabricated perfluoro functionalized CA electrospun nanofibrous mats (FS/CA-NF) with superhydrophobic character for the water/oil separation. Perfluoroalkoxysilane has been used as selective surface modifier and water repellent agent chemically reacting with the CA to form a new and novel hybrid material. Thermal treatment to cure alkoxysilane groups with available $-\mathrm{OH}$ anchors allowed the synthesis and further condensation of the components to produce novel, hybrid oil/water separator with remarkable features. Improved and modified electrospun hybrid nanofibrous mats provided remarkably robust, superhydrophobic, stiff and easy to fabricate separation systems. Conducted experiments have yielded high amount of oil/water separation even in the repetitive applications.

\section{ASSOCIATED CONTENT}

\section{S Supporting Information}

The Supporting Information is available free of charge on the ACS Publications website at DOI: 10.1021/acsami.6b05429.

Fiber diameters, FT-IR investigation, 1-H NMR results, CA-NF water adsorption and diameter plot, swelling features, EDX atomic mapping for CA-NF, repetitive contact angle measurements, EDX quantification table, thermal analysis of the components, and final material, blank separation experiments with CA (PDF)

Video showing the basic separation experiment (AVI)

\section{AUTHOR INFORMATION}

\section{Corresponding Authors}

*E-mail: arslan@unam.bilkent.edu.tr.

*E-mail: uyar@unam.bilkent.edu.tr.

\section{Author Contributions}

O.A. and Z.A. conducted experiments, and O.A., Z.A., and T.U. have been equally contributed to the written the manuscript. All authors have given approval to the final version of the manuscript.

\section{Notes}

The authors declare no competing financial interest.

\section{ACKNOWLEDGMENTS}

O.A. acknowledges TUBITAK BIDEB for 2232 Post Doc programme (project no. 115C095) and financial support. Z.A. thanks TUBITAK-BIDEB and TUBITAK (project no. 213M185) for the $\mathrm{PhD}$ scholarship.T.U. acknowledges partial support of The Turkish Academy of Sciences-Outstanding Young Scientists Award Program (TUBA-GEBIP). The authors thank to Dr. Aslı Çelebioğlu for her practical help and fruitful discussions.

\section{REFERENCES}

(1) Yoon, K.; Hsiao, B. S.; Chu, B. Functional nanofibers for environmental applications. J. Mater. Chem. 2008, 18, 5326-5334.

(2) Wendorff, J.H.; Agarwal, S.; Greiner, A. Electrospinning: Materials, Processing, and Applications; John Wiley \& Sons: Germany, 2012.

(3) Thavasi, V.; Singh, G.; Ramakrishna, S. Electrospun nanofibers in energy and environmental applications. Energy Environ. Sci. 2008, 1, 205-221.

(4) Greiner, A.; Wendorff, J. H. Electrospinning: a fascinating method for the preparation of ultrathin fibers. Angew. Chem., Int. Ed. 2007, 46, 5670-5703.

(5) Bhardwaj, N.; Kundu, S. C. Electrospinning: a fascinating fiber fabrication technique. Biotechnol. Adv. 2010, 28, 325-347.

(6) Celebioglu, A.; Sen, H. S.; Durgun, E.; Uyar, T. Molecular Entrapment of Volatile Organic Compounds (VOCs) by Electrospun Cyclodextrin Nanofibers. Chemosphere 2016, 144, 736-744.

(7) Ding, Z.; Salim, A.; Ziaie, B. Selective nanofiber deposition through field-enhanced electrospinning. Langmuir 2009, 25 (17), 9648-9652.

(8) Tchemtchoua, V. T.; Atanasova, G.; Aqil, A.; Filée, P.; Garbacki, N.; Vanhooteghem, O.; Deroanne, C.; Noël, A.; Jérome, C.; Nusgens, B.; Poumay, Y.; Colige, A. Development of a chitosan nanofibrillar scaffold for skin repair and regeneration. Biomacromolecules 2011, 12 (9), 3194-3204. 
(9) Yang, Y.; Xia, T.; Chen, F.; Wei, W.; Liu, C.; He, S.; Li, I. Electrospun fibers with plasmid bFGF polyplex loadings promote skin wound healing in diabetic rats. Mol. Pharmaceutics 2012, 9, 48-58.

(10) Senthamizhan, A.; Çelebioglu, A.; Bayir, S.; Gorur, M.; Doganci, E.; Yilmaz, F.; Uyar, T. Highly Fluorescent Pyrene-Functional Polystyrene Copolymer Nanofibers for Enhanced Sensing Performance of TNT. ACS Appl. Mater. Interfaces 2015, 7, 21038-21046.

(11) Nguyen, L. T. H.; Chen, S.; Elumalai, N. K.; Prabhakaran, M. P.; Zong, Y.; Vijila, C.; Allakhverdiev, S. I.; Ramakrishna, S. Biological, Chemical, and Electronic Applications of Nanofibers. Macromol. Mater. Eng. 2013, 298, 822-867.

(12) Ma, Z.; Kotaki, M.; Ramakrishna, S. Electrospun cellulose nanofiber as affinity membrane. J. Membr. Sci. 2005, 265, 115-127.

(13) Celebioglu, A.; Demirci, S.; Uyar, T. Cyclodextrin-grafted electrospun cellulose acetate nanofibers via "Click" reaction for removal of phenanthrene. Appl. Surf. Sci. 2014, 305, 581-588.

(14) Demirci, S.; Celebioglu, A.; Uyar, T. Surface modification of electrospun cellulose acetate nanofibers via RAFT polymerization for DNA adsorption. Carbohydr. Polym. 2014, 113, 200-207.

(15) Habibi, Y.; Lucia, L. A.; Rojas, O. J. Cellulose nanocrystals: chemistry, self-assembly, and applications. Chem. Rev. 2010, 110, 3479-3500.

(16) Munaweera, I.; Aliev, A.; Balkus, K. J., Jr. Electrospun Cellulose Acetate-Garnet Nanocomposite Magnetic Fibers for Bioseparations. ACS Appl. Mater. Interfaces 2014, 6, 244-251.

(17) Chattopadhyay, S.; Hatton, T. A.; Rutledge, G. C. Aerosol filtration using electrospun cellulose acetate fibers. J. Mater. Sci. 2016, 51, 204-217.

(18) Duarte, A. P.; Cidade, M. T.; Bordado, J. C. Cellulose acetate reverse osmosis membranes: optimization of the composition. J. Appl. Polym. Sci. 2006, 100, 4052-4058.

(19) Ganesh, V. A.; Ranganath, A. S.; Sridhar, R.; Raut, H. K.; Jayaraman, S.; Sahay, R.; Ramakrishna, S.; Baji, A. Cellulose AcetatePoly(N-isopropylacrylamide)-Based Functional Surfaces with Temperature- Triggered Switchable Wettability. Macromol. Rapid Commun. 2015, 36, 1368-1373.

(20) Tian, Y.; Wu, M.; Liu, R.; Li, Y.; Wang, D.; Tan, J.; Wu, R.; Huang, Y. Electrospun membrane of cellulose acetate for heavy metal ion adsorption in water treatment. Carbohydr. Polym. 2011, 83, 743748.

(21) Celebioglu, A.; Uyar, T. Electrospun porous cellulose acetate fibers from volatile solvent mixture. Mater. Lett. 2011, 65, 2291-2294.

(22) Jayalakshmi, A.; Kim, I.; Kwon, Y. Cellulose acetate graft(glycidylmethacrylate- g-PEG) for modification of AMC ultrafiltration membranes to mitigate organic fouling. RSC Adv. 2015, 5, 4829048300 .

(23) Achoundong, C.S. K.; Bhuwania, N.; Burgess, S. K.; Karvan, O.; Johnson, J. R; Koros, W. J. Silane modification of cellulose acetate dense films as materials for acid gas removal. Macromolecules 2013, 46, 5584-5594.

(24) Feng, Q.; Hou, D.; Zhao, Y.; Xu, T.; Menkhaus, T. J.; Fong, H. Electrospun regenerated cellulose nanofibrous membranes surfacegrafted with polymer chains/brushes via the atom transfer radical polymerization method for catalase immobilization. ACS Appl. Mater. Interfaces 2014, 6, 20958-2096.

(25) Senthamizhan, A.; Balusamy, B.; Celebioglu, A.; Uyar, T. Nanotraps" in porous electrospun fibers for effective removal of lead(II) in water. J. Mater. Chem. A 2016, 4, 2484.

(26) Danks, A. E.; Hall, S. R.; Schnepp, Z. The evolution of 'sol-gel' chemistry as a technique for materials synthesis. Mater. Horiz. 2016, 3, 91-112.

(27) Karatum, O.; Steiner, S. A., III; Griffin, J. S.; Shi, W.; Plata, D. L. Flexible, Mechanically Durable Aerogel Composites for Oil Capture and Recovery. ACS Appl. Mater. Interfaces 2016, 8, 215-224.

(28) Ganesh, V. A.; Ranganath, A. S.; Baji, A.; Wong, H. C.; Raut, H. K.; Sahay, R.; Ramakrishna. Electrospun Differential Wetting Membranes for Efficient Oil-Water Separation, S. Macromol. Mater. Eng. 2016, 301, 812.
(29) Li, J.; Zhou, Y.; Luo, Z. Smart Fiber Membrane for pH-Induced Oil/Water Separation. ACS Appl. Mater. Interfaces 2015, 7, 1964319650.

(30) Zhang, C.; Li, P.; Cao, B. Electrospun Microfibrous Membranes Based on PIM- 1/POSS with High Oil Wettability for Separation of Oil-Water Mixtures and Cleanup of Oil Soluble Contaminants. Ind. Eng. Chem. Res. 2015, 54, 8772-8781.

(31) Fang, W.; Liu, L.; Li, T.; Dang, Z.; Qiao, C.; Xu, J.; Wang, Y. Electrospun N-Substituted Polyurethane Membranes with SelfHealing Ability for Self-Cleaning and Oil/Water Separation. Chem. Eur. J. 2016, 22, 878-883.

(32) Yi, D.; Xu, C.; Tang, R.; Zhang, X.; Caruso, F.; Wang, Y. Synthesis of Discrete Alkyl-Silica Hybrid Nanowires and Their Assembly into Nanostructured Superhydrophobic Membranes. Angew. Chem. 2016, 128, 8515-8520.

(33) Zhang, J.; Seeger, S. Polyester Materials with Superwetting Silicone Nanofi laments for Oil/Water Separation and Selective Oil Absorption. Adv. Funct. Mater. 2011, 21, 4699-4704.

(34) Wang, X.; Xu, S.; Tan, Y.; Du, J.; Wang, J. Synthesis and characterization of a porous and hydrophobiccellulose-based composite for efficient and fast oil-water separation. Carbohydr. Polym. 2016, 140, 188-194.

(35) Arslan, O.; Arpac, E.; Sayllkan, H. Siliconcarbide embedded hybrid nanocomposites as abrasion resistant coating. J. Inorg. Organomet. Polym. Mater. 2010, 20, 284-292.

(36) Arslan, O.; Arpaç; Sayllkan, F.; Sayılkan, H. Hybrid sol-gel coating on Al. J. Mater. Sci. 2007, 42, 2138-2142.

(37) Schmidt, H.; Kasemann, R. Eur Patent EP0587667 B1, 1994.

(38) Çamurlu, H. E.; Mathur, S.; Arslan, O.; Akarsu, E. Modification of Hexagonal Boron Nitride Nanoparticles with Fluorosilane. Ceram. Int. 2016, 42, 6312-6318.

(39) Lim, J. M.; Moon, J. H.; Yi, G.; Heo, C.; Yang, S. Fabrication of one-dimensional colloidal assemblies from electrospun nanofibers. Langmuir 2006, 22 (8), 3445-3449.

(40) Hou, Z.; Li, G.; Lian, H.; Lin, J. One- dimensional luminescent materials derived from the electrospinning process: preparation, characteristics and application. J. Mater. Chem. 2012, 22, 5254-5276.

(41) Lu, X.; Wang, C.; Wei, Y. One-dimensional composite nanomaterials: synthesis by electrospinning and their applications. Small 2009, 5 (21), 2349-2370.

(42) Pereira, C.; Alves, C.; Monteiro, A.; Magén, C.; Pereira, A. M.; Ibarra, A.; Ibarra, M. R.; Tavares, P. B.; Araújo, J. P.; Blanco, G.; Pintado, J. M.; Carvalho, A. P.; Pires, J.; Pereira, M. F. R.; Freire, C. Designing Novel Hybrid Materials by One-Pot Co-condensation: From Hydrophobic Mesoporous Silica Nanoparticles to Superamphiphobic Cotton Textiles. ACS Appl. Mater. Interfaces 2011, 3, 2289-2299.

(43) Bellanger, H.; Darmanin, T.; Taffin de Givenchy, E.; Guittard, F. Chemical and Physical Pathways for the Preparation of Superoleophobic Surfaces and Related Wetting Theories. Chem. Rev. 2014, 114 (5), 2694-2716.

(44) Ferrero, F.; Periolatto, M. Application of fluorinated compounds to cotton fabrics via sol-gel Applied Surface. Appl. Surf. Sci. 2013, 275, 201-207.

(45) Nguyen, S. T.; Feng, J.; Le, N. T.; Le, A.T. T.; Hoang, N.; Tan, V. B. C.; Duong, H. M. Preparation of Superhydrophobic Magnetic Cellulose Sponge for Removing Oil from Water. Ind. Eng. Chem. Res. 2013, 52, 18386-18391.

(46) Michielsen, S.; Lee, H. J. Design of a superhydrophobic surface using woven structures. Langmuir 2007, 23, 6004-6010.

(47) Vasiljevic, J.; Gorjanc, M.; Tomsic, B.; Orel, B.; Jerman, I.; Mozetic, M.; Vesel, A.; Simoncic, B. The surface modification of cellulose fibres to create super-hydrophobic, oleophobic and selfcleaning properties. Cellulose 2013, 20, 277-289.

(48) Cunha, A. G.; Gandini, A. Turning polysaccharides into hydrophobic materials: a critical review. Part 1. Cellulose 2010, 17, 875-889. 
(49) Yong, J.; Chen, F.; Yang, Q.; Bian, H.; Du, G.; Shan, C.; Huo, J.; Fang, Y.; Hou, X. Oil-Water Separation: Oil-Water Separation: A Gift from the Desert. Adv. Mater. Interfaces 2016, 3, 1500650.

(50) Hayase, G.; Kanamori, K.; Fukuchi, M.; Kaji, H.; Nakanishi, K. Facile Synthesis of Marshmallow-like Macroporous Gels Usable under Harsh Conditions for the Separation of Oil and Water. Angew. Chem., Int. Ed. 2013, 52, 1986-1989.

(51) Wang, G.; Zeng, Z.; Wang, H.; Zhang, L.; Sun, X.; He, Y.; Li, L.; Wu, X.; Ren, T.; Xue, Q. Low Drag Porous Ship with Superhydrophobic and Superoleophilic Surface for Oil Spills Cleanup. ACS Appl. Mater. Interfaces 2015, 7, 26184-26194.

(52) Kim, D. H.; Jung, M. C.; Cho, S.; Kim, S. H.; Kim, H.; Lee, H. J.; Oh, K. H.; Moon, M. UV- responsive nano-sponge for oil absorption and desorption. Sci. Rep. 2015, 5, 12908.

(53) Cervin, N. T.; Aulin, C.; Larsson, P. T.; Wagberg, L. Ultra porous nanocellulose aerogels as separation medium for mixtures of oil/water liquids. Cellulose 2012, 19, 401-410. 